\title{
Histopathologic, immunophenotypic and cytogenetic features of pulmonary mucoepidermoid carcinoma
}

\author{
Anja C Roden ${ }^{1}$, Joaquín J García ${ }^{1}$, Rebecca N Wehrs ${ }^{1}$, Thomas V Colby ${ }^{2}$, Andras Khoor $^{3}$, \\ Kevin O Leslie ${ }^{2}$ and Longwen Chen ${ }^{2}$ \\ ${ }^{1}$ Department of Laboratory Medicine \& Pathology, Mayo Clinic, Rochester, MN, USA; ${ }^{2}$ Department of \\ Laboratory Medicine \& Pathology, Mayo Clinic, Arizona, AZ, USA and ${ }^{3}$ Department of Laboratory Medicine \\ \& Pathology, Mayo Clinic, Jacksonville, FL, USA
}

\begin{abstract}
Pulmonary mucoepidermoid carcinoma is an uncommon but distinctive manifestation of mucoepidermoid carcinoma. Pulmonary mucoepidermoid carcinoma occurs in adults and children and can cause diagnostic problems, especially in small biopsies. Few studies have characterized the histologic and immunophenotypic features of pulmonary mucoepidermoid carcinoma. $t(11 ; 19)(q 21 ; p 13)$ is considered disease-defining for mucoepidermoid carcinoma; its significance in pulmonary mucoepidermoid carcinoma warrants further study. Forty three pulmonary mucoepidermoid carcinomas were re-reviewed and graded according to the Brandwein grading system for mucoepidermoid carcinoma. Four cases were excluded because of a split opinion between pathology report and re-review. These cases were negative for MAML2 rearrangement by FISH. TTF-1, napsin A, p40 and p63 immunostains were scored: 0 (negative), 1 (1-25\% tumor cells), 2 (26-50\%), $3(51-75 \%)$ or 4 ( $>75 \%$ ). FISH to detect MAML2 rearrangement used a MAML2-11q21 break-apart probe. Thirty nine pulmonary mucoepidermoid carcinoma (4 low, 30 intermediate, 5 high grade) contained mucous, epidermoid and intermediate cells and lacked keratinization and in situ carcinoma of the overlying epithelium. All cases with available gross description $(n=22)$ had a central/endo- or peribronchial location. All 25 cases tested for immunohistochemistry were positive (scores 1-4) for p63; 23 also expressed p40. In six cases, the p63 score was higher than p40. TTF-1 and napsin were uniformly negative in all 25 cases. MAML2 rearrangement was identified by FISH in each of the 24 cases tested ( 3 low, 19 intermediate, 2 high grade). Clinical history was available in 29 patients (15 men) (median age, 48 years) with follow-up in 24 (median, 8.4 years). Five patients died of unrelated causes; one developed metastatic pulmonary mucoepidermoid carcinoma. In conclusion, features helpful in distinguishing pulmonary mucoepidermoid carcinoma from other lung cancers include its central/endo- or peribronchial location together with the presence of mucous cells, p63 expression, lack of keratinization and MAML2 rearrangement. TTF-1 and napsin are typically not expressed.

Modern Pathology (2014) 27, 1479-1488; doi:10.1038/modpathol.2014.72; published online 18 April 2014
\end{abstract}

Keywords: MAML2 rearrangement; napsin; p40; p63; primary pulmonary mucoepidermoid carcinoma; TTF-1

Primary pulmonary mucoepidermoid carcinomas are rare neoplasms that account for $9 \%$ of malignant lung tumors in children ${ }^{1}$ and $<0.5 \%$ of all lung tumors in adults. ${ }^{2}$ They are usually centrally located and are presumed to originate from submucosal

Correspondence: Dr AC Roden, MD, Division of Anatomic Pathology, Mayo Clinic Rochester, Hilton 11, 200 First Street SW, Rochester, MN 55905, USA.

E-mail: Roden.anja@mayo.edu

Presented as abstract at the Annual Meeting of the United States \& Canadian Academy of Pathology, March 2014, San Diego, CA, USA.

Received 25 February 2014; revised 21 March 2014; accepted 22 March 2014; published online 18 April 2014 minor salivary-type glands in large airways. However, distinguishing pulmonary mucoepidermoid carcinoma from other primary lung nonsmall cell carcinoma has potential prognostic and therapeutic implications. ${ }^{3-5}$ Few studies have attempted to characterize the histologic and immunophenotypic features of pulmonary mucoepidermoid carcinoma.

There is a strong association between mucoepidermoid carcinoma and $t(11 ; 19)(q 21 ; p 13) .{ }^{6,7}$ The most common genes involved in this translocation are CREB Regulated Transcription Coactivator1 (CRCT1, also known as MECT1, TORC1 or WAMTP1) on chromosome $19 \mathrm{p} 13$ and a member of the 
MasterMind-Like gene family, MAML2, on chromosome 11q21. In salivary gland tumors, MAML2 rearrangement has been identified in $40-75 \%$ of mucoepidermoid carcinomas but not in other salivary gland tumors. ${ }^{8-10}$ Furthermore, Clauditz et al ${ }^{8}$ demonstrated that the frequency of MAML2 rearrangement significantly decreased with increased tumor grade. Achcar et al ${ }^{11}$ showed MAML2 rearrangement in 13 of $17(77 \%)$ pulmonary mucoepidermoid carcinomas, including 10 (of 10) low-grade and 3 (of 7) high-grade pulmonary mucoepidermoid carcinomas, while other lung tumors including adenosquamous, squamous and primary lung adenocarcinomas did not harbor that translocation. As the $\mathrm{t}(11 ; 19)(\mathrm{q} 21 ; \mathrm{p} 13)$ translocation is considered disease-defining for mucoepidermoid carcinoma, its significance in pulmonary mucoepidermoid carcinoma warrants further study.

We performed fluorescence in situ hybridization (FISH) for the detection of MAML2 rearrangement and characterized a large number of pulmonary mucoepidermoid carcinomas histologically and immunophenotypically.

\section{Materials and methods}

\section{Patients and Specimens}

Pathology and consultation files at Mayo Clinic Arizona, Jacksonville and Rochester (1972-2013) were searched for cases of pulmonary mucoepidermoid carcinoma. All available medical records were reviewed.

The study was approved by the Mayo Clinic Institutional Review Board (IRB\# 12-008277).

\section{Histopathologic Criteria}

Hematoxylin and eosin-stained slides from all selected patients were re-reviewed independently by two surgical pathologists (ACR, JJG) who were blinded to clinical information and immunopathologic and cytogenetic results. Disagreements were solved by consensus. A total of 21 (of 43) identified cases were previously reported., ${ }^{4,12}$ In all cases, clinical and radiologic features of the tumor were suggestive of lung origin. Cases with morphologic features consistent with mucoepidermoid carcinoma were included as study cases. Mucoepidermoid carcinomas were morphologically defined by the presence of variable proportions of epidermoid, mucous and intermediate cells in the absence of keratinization or in situ carcinoma of the surface epithelium as described in the current WHO classification of pulmonary mucoepidermoid carcinoma. ${ }^{13}$ All cases were graded according to the criteria described by Brandwein et al. ${ }^{3}$ If the opinion about the diagnosis of pulmonary mucoepidermoid carcinoma differed between the original pathology report and the reviewers, morphology and positive FISH results were required for inclusion of the case.

\section{Immunohistochemical Staining and Scoring}

Formalin-fixed paraffin-embedded tissue blocks were sectioned at $4 \mu \mathrm{m}$. Slides were stained with hematoxylin-eosin and consecutive slides were stained with antibodies against TTF-1 (clone SPT24, Leica Microsystems, Buffalo Grove, IL, USA), napsin A (clone IP64, Leica Microsystems), p40 (polyclonal, Biocare Medical, Concord, CA, USA) and p63 (clone BC4A4, Biocare Medical).

Staining was scored by one surgical pathologist (ACR) as follows: 0 (negative), $1(1-25 \%$ tumor cell staining), 2 (26-50\%), 3 (51-75\%), and 4 (>75\%).

\section{FISH for MAML2 Rearrangement}

FISH for MAML2 rearrangement was performed as previously described ${ }^{14}$ using a break-apart probe. In short, MAML2 break-apart probe consisted of bacterial artificial chromosomes (BAC) (Children's Hospital Research Institute, Oakland, CA, USA, or Life Technologies, Grand Island, NY, USA) flanking the $5^{\prime}$ and $3^{\prime}$ sides of MAML2. $5^{\prime}$ BACs were labeled in SpectrumOrange (Abbott Laboratories, Abbott Park, IL, USA) and consisted of RP11-1056O10, CTD2325K3, and RP11-8N17. 3' BACs were labeled in SpectrumGreen (Abbott Laboratories) and consisted of CTD-2252L1, RP11-123F20, RP11-7D4, and CTD254417. A total of 200 tumor cells were counted and percent split signal was recorded. Three cases of salivary gland mucoepidermoid carcinoma were used for positive control and normal salivary (parotid) gland tissue was used for negative control.

\section{Results}

Cases Excluded on the Basis of Split Opinion Between Original Pathology Report and Reviewers Diagnosis

Forty three cases of pulmonary mucoepidermoid carcinoma were identified in the pathology and consultation files. Upon re-review, there was a split opinion about the diagnosis of pulmonary mucoepidermoid carcinoma in five cases. These cases included a $0.4 \mathrm{~cm}$ endobronchial lesion that morphologically resembled a benign minor salivary gland lesion, an epithelioid neoplasm with cribriform architecture most consistent with an adenocarcinoma, a neoplasm with a prominent spindle cell component, and a case that morphologically resembled a cystadenocarcinoma (Figure 1). In addition, MAML2 rearrangement was identified in only one of the five cases. These four MAML2negative morphologically atypical cases were excluded from further analysis. Therefore, the study population comprised 39 pulmonary mucoepidermoid carcinomas. 


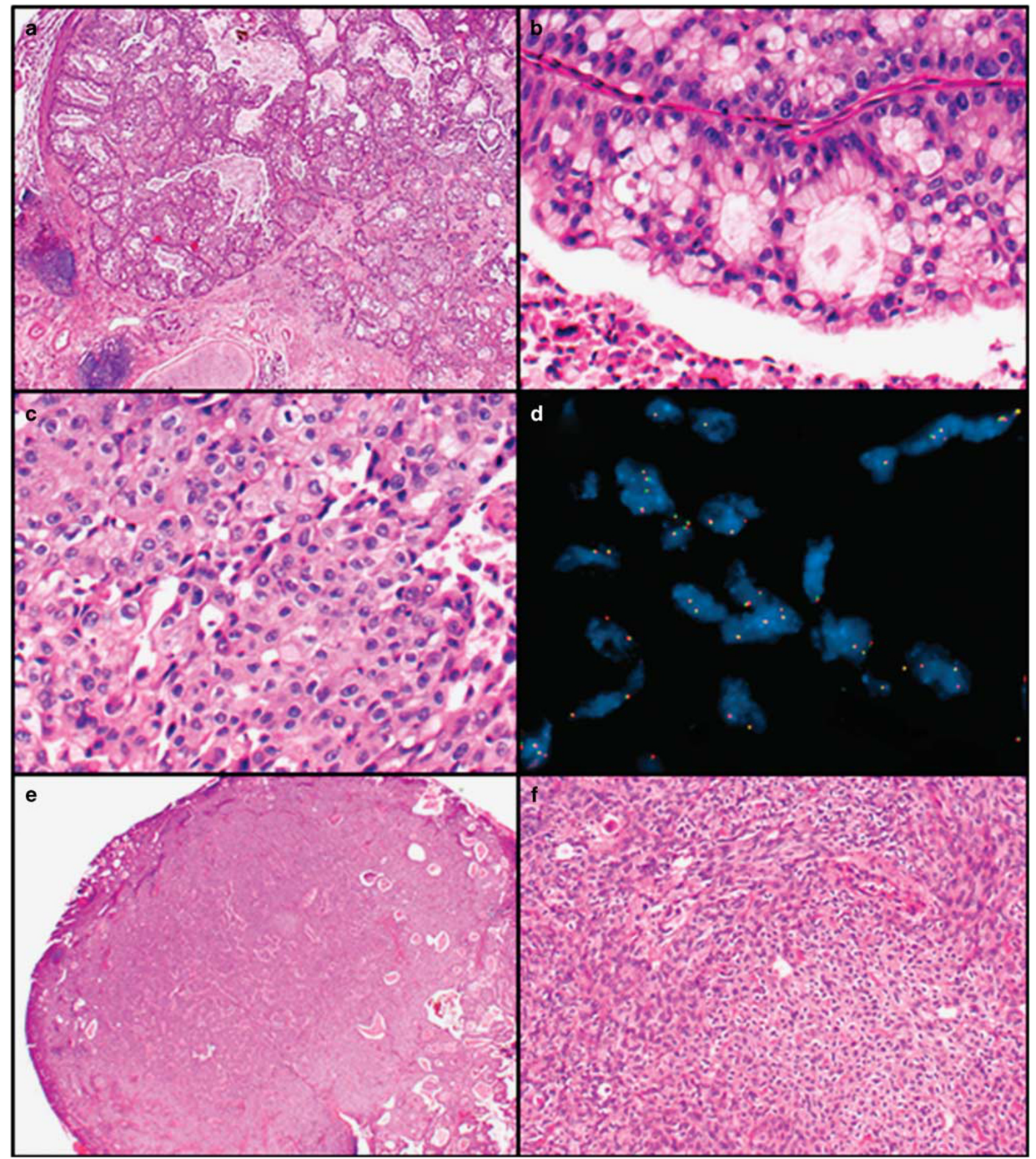

Figure 1 Cases thought not to represent mucoepidermoid carcinoma morphologically upon re-review; each of these cases showed an intact MAML2 locus by FISH and was excluded from the study. Moderately differentiated adenocarcinoma, NOS (not otherwise specified) (a-d). Low power view shows an invasive neoplasm in the vicinity to a large airway that comprises a complex glandular architecture-note cartilage (a). The glands are lined by mucin-containing epithelial cells (b). One area comprises nests of cohesive epithelial cells without gland formation (c). FISH studies of the tumor indicate an intact MAML2 locus (colocalization of the red and green signals produces a yellow color and indicates an intact locus) (d). Of note, this patient also has a history of colonic adenocarcinoma. Spindle cell neoplasm (e-f). Low magnification reveals nests and sheets of neoplastic cells (e). At high power, there are nests of bland-appearing spindle cells intermixed with foci of more round to oval cells (f). Papillary cystadenocarcinoma (g-h). This centrally located tumor comprises variably sized cystic spaces (g), many of which contain intraluminal papillary growth; however, epidermoid and intermediate cells are not identified (h). Benign submucosal lesion (i, j). This small submucosal nodule (i) comprises short fascicles of benign appearing spindle cells, possibly fibroblasts or myofibroblasts intermixed with collagen fibrosis and ductular proliferation. However, there are no epidermoid, mucous or intermediate cells identified. Magnification: $\times 20$ (a, e, g), $\times 400$ (b,c), 1000 oil immersion $(\mathbf{d}), \times 200(\mathbf{f}, \mathbf{j}), \times 100(\mathbf{h}), \times 40(\mathbf{i})$ 


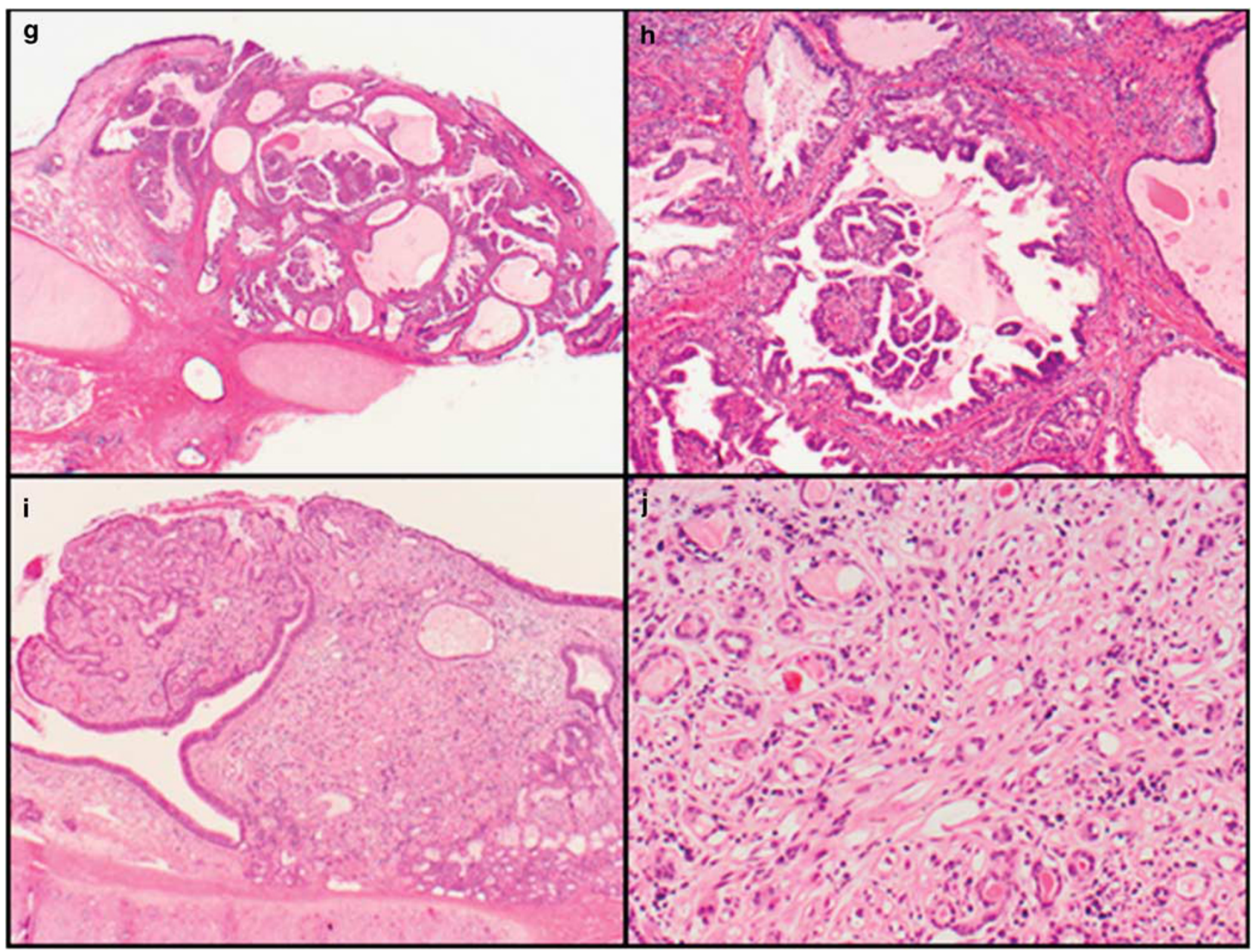

Figure 1 Continued.

\section{Morphologic Characteristics of Primary Pulmonary Mucoepidermoid Carcinoma}

According to the morphologic definition of mucoepidermoid carcinoma, ${ }^{13}$ all cases contained a variable number of mucus-secreting, epidermoid and intermediate cells. Tumor grades and morphologic features are summarized in Table 1. The vast majority of pulmonary mucoepidermoid carcinomas were classified as intermediate grade, most commonly because of tumor invasion in small nests and islands (Figure 2). No tumor showed in situ carcinoma of the overlying mucosal surface. TTF-1 and napsin were not expressed in any of the 26 cases tested.

\section{All Primary Pulmonary Mucoepidermoid Carcinoma Tested Expressed p63}

Tissue blocks were available in 26 cases. In one case there was no staining with any of the antibodies tested and the internal control for p63 and p40 was negative. Therefore, this case was excluded from the evaluation of immunophenotype.
Results of immunostains are summarized in Table 1. In cases with concordant p63 and p40 staining $(n=19)$, low-grade pulmonary mucoepidermoid carcinomas exhibited scores of $1(n=3)$ and 3 $(n=1)$, intermediate-grade pulmonary mucoepidermoid carcinomas showed scores of $1(n=6), 2$ $(n=3)$ and $4 \quad(n=4)$ and high-grade pulmonary mucoepidermoid carcinomas had scores of 3 $(n=1)$ and $4(n=1)$. In six cases p63 scores were higher than the respective p40 scores; these cases had p63 score of $1(n=5)$ or $2(n=1)$ (Figure 2). Overall, two cases had a p63 score of 1 but were negative by $\mathrm{p} 40$. No case had a higher score for $\mathrm{p} 40$ than p63. All cases with discordant p63-p40 staining were classified as intermediate grade.

\section{MAML2 Rearrangement was Identified in all Primary Pulmonary Mucoepidermoid Carcinoma Tested}

MAML2 rearrangement was identified in low-grade $(n=3)$, intermediate-grade $(n=19)$, and high-grade $(n=2)$ pulmonary mucoepidermoid carcinomas (Table 1; Figure 3). The median percent split signal 
Table 1 Morphologic, immunophenotypic and cytogenetic features of pulmonary mucoepidermoid carcinoma

\begin{tabular}{l} 
Characteristic \\
\hline Morphologic features $(\mathrm{n}=39)$ \\
Tumor histologic grade (Brandwein) $(n=39)$ \\
Low \\
Intermediate \\
High \\
Invasion in small nests and islands \\
(evaluable, $n=38$ ) \\
Intracystic component $<25 \%$ \\
Pronounced nuclear atypia \\
Perineural invasion \\
Tumor-associated lymphoid proliferation \\
Keratinization \\
In situ carcinoma of the overlying epithelium
\end{tabular}

$\mathrm{N}(\%)$

Anatomic location $(\mathrm{n}=22)$
Central/endobronchial

Peribronchial

Immunophenotypic features $(\mathrm{n}=25)$

p63 and p40 with concordant staining pattern

Score 1

Score 2

Score 3

Score 4

p63 score $=$ p40 score +1

p63 score $=$ p40 score +2

TTF-1 and/or napsin expression

Cytogenetic Features $(\mathrm{n}=24)$

MAML2 rearrangement
$21(95.5)$

$1(0.5)$

$4(10.3)$

5 (12.8)

$33(86.8)$

7 (17.9)

$1(2.6)$

$1(2.6)$

$29(74.4)$

0

0

19 (76.0)

9

3

2

5

$5(20.0)$

$1(4.0)$

0

$24(100)$
$30(76.9)$

was $47 \%$ (range, $11-87 \%$ ). In two additional cases, hybridization failed, one of which also lacked expression of internal control by immunostains. None of the cases tested for MAML2 rearrangement showed an intact probe.

\section{Clinical Information and Follow-Up of Patients}

Clinical history was available in 29 cases (15 men) of the study set. The median age at tissue diagnosis was 47.6 years (range, 6.1-75.3 years). Twenty seven patients underwent complete resection, two had a biopsy. In 21 of 27 resection cases with available information lymph nodes were sampled. In one case (of $21,4.8 \%$ ), a single intraparenchymal peribronchial (IPPB) lymph node was positive for metastatic pulmonary mucoepidermoid carcinoma. This pulmonary mucoepidermoid carcinoma was of intermediate histologic grade.

Follow-up was available in 24 patients of the study set with a median follow-up time of 8.4 years (range, 0.2-31.5 years). Five patients died of unrelated causes. One (of 24, 4.2\%) patient developed a liver metastasis 15 months after complete resection of an intermediate-grade pulmonary mucoepidermoid carcinoma. The metastasis was treated with stereotactic body radiation therapy. Four years later the liver metastasis recurred and was treated with percutaneous radiofrequency ablation. The patient is alive without evidence of disease 5.6 years following the initial tumor resection. No patient died of disease. Only one patient with high-grade pulmonary mucoepidermoid carcinoma had followup available and is alive without evidence of disease 4.1 years following resection. The patient with a positive lymph node at the time of resection died 4 years later due to an unrelated disease without evidence of recurrence or metastasis.

All four patients that were excluded from the study based on atypical histologic features and absence of MAML2 rearrangement underwent complete resection. Tumor sizes ranged from 0.4 to $2.2 \mathrm{~cm}$. In all cases, lymph nodes were sampled during surgery and two IPPB lymph nodes were positive in one case (of $4,25 \%$ ). In that particular case, pleural metastases were also identified. This patient died 8 months after surgery due to disease. Another patient died 10 years after surgery, likely due to an unrelated disease because at his last follow-up 11 months before his death there was no evidence of recurrence or metastasis. The other two patients were lost to follow-up 6 years or 6 months after surgery without evidence of disease.

\section{Discussion}

Herein we characterize morphologic, immunophenotypic and cytogenetic features of a large number of primary pulmonary mucoepidermoid carcinoma.

Although most commonly identified in the head and neck, mucoepidermoid carcinomas can occur in many sites of the body including breast, ${ }^{15}$ lung, ${ }^{16}$ skin, ${ }^{17}$ and thymus ${ }^{18}$ among others. Independent of the site of the tumor, mucoepidermoid carcinomas are composed of epidermoid, mucous and intermediate cells, and lack keratinization and in situ carcinoma of the overlying mucosal surface. Accordingly, all cases in the current study comprised a variable proportion of these three cell types and none showed any keratinization or in situ carcinoma of the overlying epithelium.

Although the morphologic distinction of pulmonary mucoepidermoid carcinoma from its mimics such as adenosquamous carcinoma, squamous cell carcinoma and adenocarcinoma can sometimes be challenging particularly in small biopsies and highgrade tumors, the separation is critical because the prognosis of mucoepidermoid carcinoma is usually superior to its mimics. For instance, in a recent study on head and neck mucoepidermoid carcinoma, 46 cases were originally identified as mucoepidermoid carcinoma in the pathology files; 24 of these cases were re-diagnosed as another tumor based on applying strict morphologic criteria, as also described in our study. ${ }^{5}$ In that study, almost all re-diagnosed cases were of a more aggressive tumor 


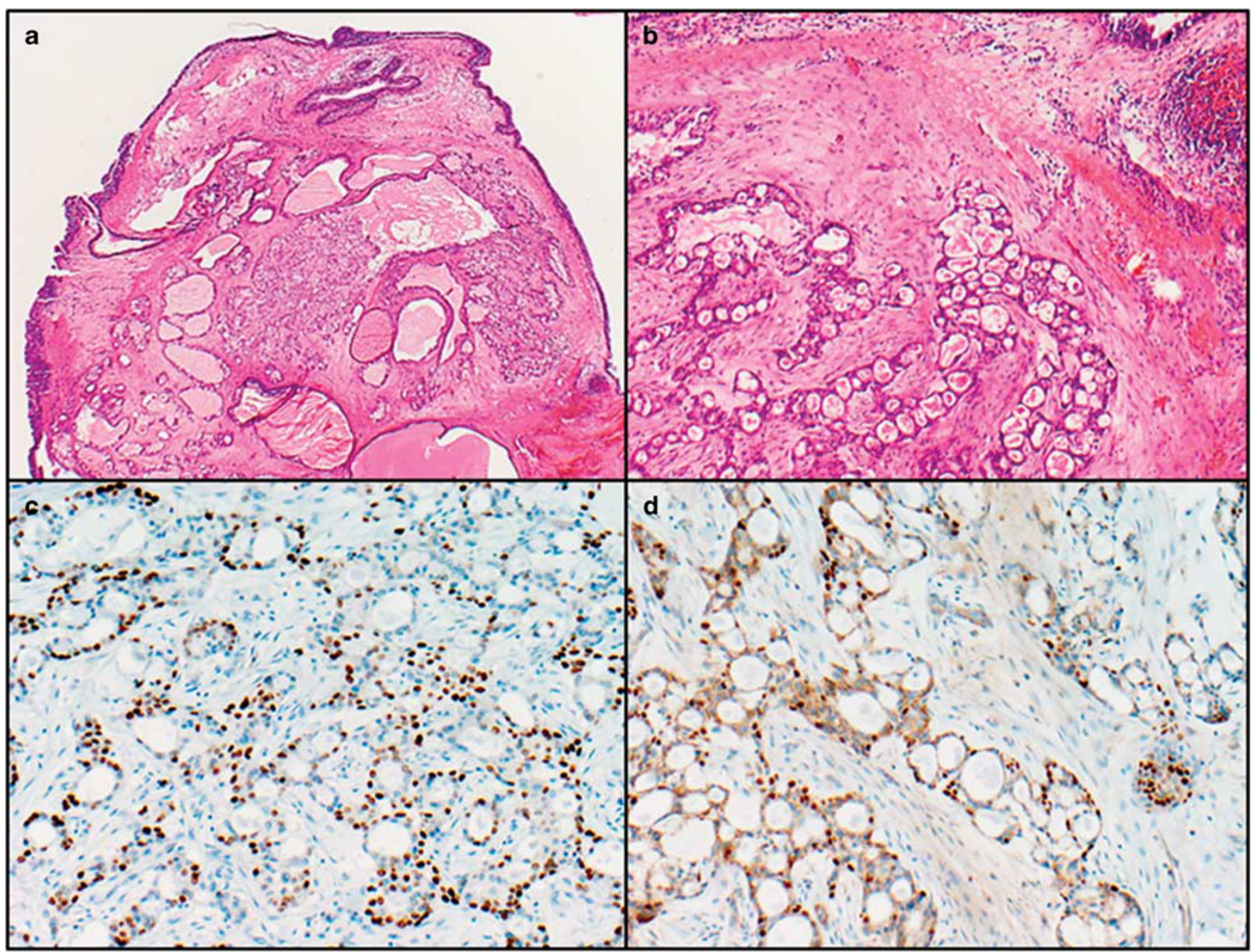

Figure 2 Intermediate grade mucoepidermoid carcinoma. This mucoepidermoid carcinoma is a centrally located tumor-note location in submucosa of a bronchus - with a cystic component that comprises $>25 \%$ of the tumor (a) and invades in small nests (b). p63 marks $\sim 60 \%$ of the tumor cells (score 3) (c), while p40 highlights $<25 \%$ of tumor cells (score 1) (d). Magnification $\times 20(\mathbf{a}), \times 100(\mathbf{b}), \times 200$ (c,d).

type, most commonly adenosquamous carcinoma, squamous cell carcinoma and salivary duct carcinoma, occurred predominantly in elderly men and were characterized by increased lymph node metastases, perineural invasion and shorter survival. In our series, no study patient died of disease, whereas one of the four excluded patients died of disease. These results emphasize the importance of applying strict morphologic criteria to diagnose pulmonary mucoepidermoid carcinoma because of prognostic and therapeutic implications.

Evidence suggests that grading of mucoepidermoid carcinoma is also significant for prognosis and management of these patients. For instance, it has recently been shown that high-grade pulmonary mucoepidermoid carcinoma have a significantly worse overall and disease-free survival than low and intermediate-grade pulmonary mucoepidermoid carcinoma. ${ }^{19}$ Furthermore, in a study of 2400 parotid mucoepidermoid carcinomas, the 5-year disease-specific survival rates were significantly different for low-grade, intermediate-grade, and high-grade mucoepidermoid carcinomas and were $98.8,97.4$, and $67.0 \%$, respectively. ${ }^{20}$ In that study, high-grade morphology was a negative prognostic factor and those tumors were more likely to have lymph node metastases than low- and intermediategrade mucoepidermoid carcinomas. In our study, only one patient with intermediate-grade morphology developed a metastasis and another patient with intermediate grade tumor had a positive lymph node at resection. Moreover, none of the low-grade pulmonary mucoepidermoid carcinomas had positive lymph nodes, metastases or recurrences. However, our study included only a few low- and high-grade pulmonary mucoepidermoid carcinomas and therefore, it is difficult to come to a respective strong conclusion.

In our study, most pulmonary mucoepidermoid carcinomas (over $75 \%$ ) were of intermediate grade, 


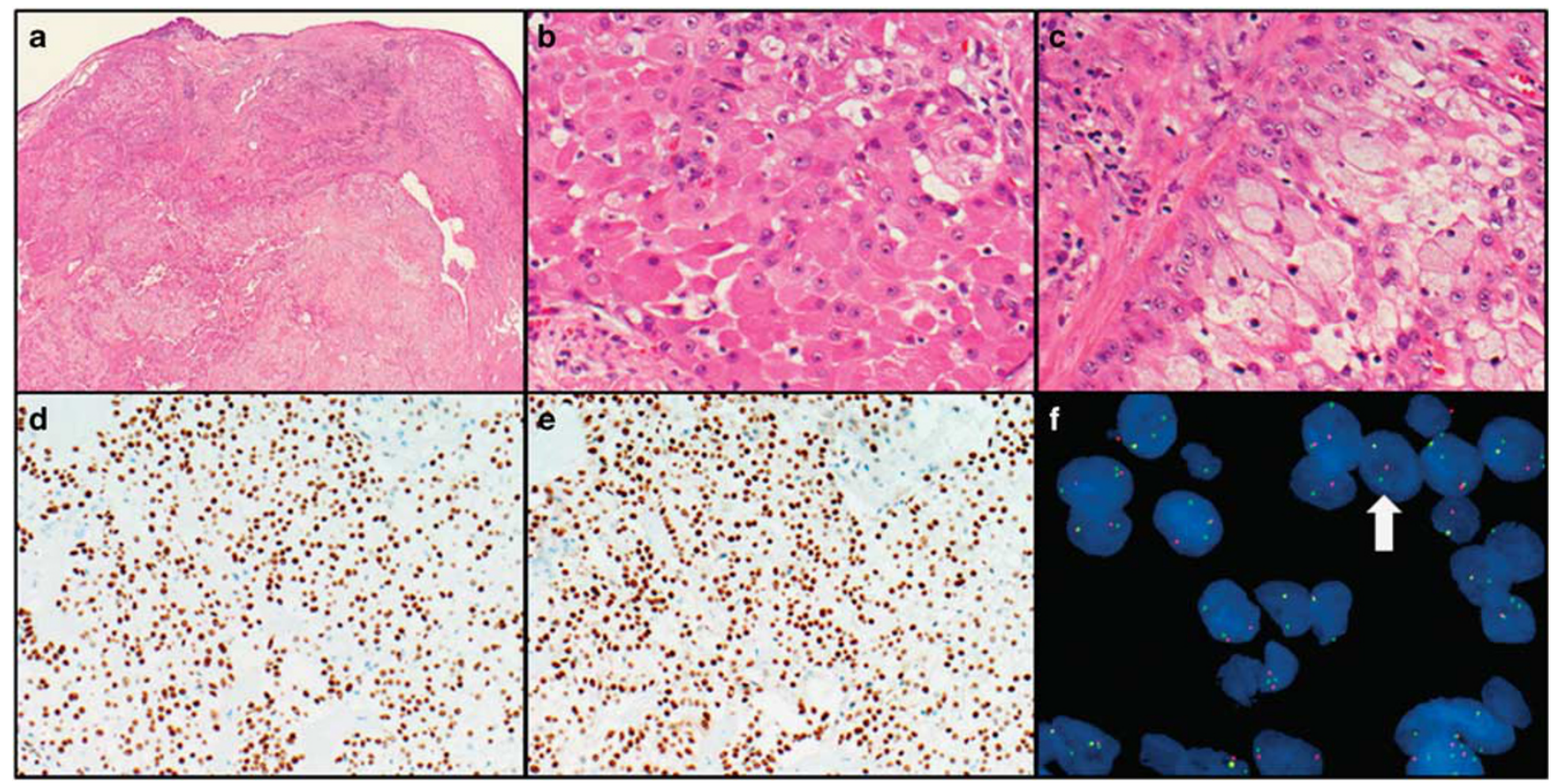

Figure 3 High-grade pulmonary mucoepidermoid carcinoma. At low magnification, nests of tumor cells in the submucosa of a large airway are growing in an invasive pattern in a background of fibrosis (a). At high power there are areas with squamous (b) and mucinous differentiation together with intermediate cells (c). The neoplastic cells strongly and diffusely express p63 (d) and p40 (e) (score 4). MAML2 locus is rearranged as evidenced by FISH (disruption of the red and green signals, arrow) (f). Magnification $\times 20$ (a), $\times 400(\mathbf{b}, \mathbf{c})$, $\times 200(\mathbf{d}, \mathbf{e}), \times 1000$ oil immersion $(\mathbf{f})$.

Table 2 Immunophenotype of pulmonary mucoepidermoid carcinoma and its morphologic mimics

\begin{tabular}{|c|c|c|c|c|}
\hline \multirow[b]{2}{*}{ Tumor type } & \multicolumn{4}{|c|}{ Immunostain ( $\%$ positive cases) } \\
\hline & p63 & $p 40$ & $T T F-1^{a}$ & Napsin \\
\hline Pulmonary mucoepidermoid carcinoma ${ }^{23,24}$ & $100^{\mathrm{b}}$ & $92^{\mathrm{b}}$ & $0^{\mathrm{b}}$ & $0^{\mathrm{b}}$ \\
\hline Squamous cell carcinoma, lung ${ }^{30-35}$ & $95-100$ & 100 & 4 & 0 \\
\hline Pulmonary adenocarcinoma $30-34,36$ & $18-34$ & $0-3^{\mathrm{C}}$ & $60-90$ & $83-87$ \\
\hline
\end{tabular}

${ }^{\mathrm{a} C l o n e ~ S P T 24 . ~}$

${ }^{\mathrm{b}}$ Results from the present study.

${ }^{\mathrm{c}} \mathrm{p} 40$ positive in $\leq 5 \%$ tumor cells.

predominantly because of tumor invasion in small nests and islands but occasionally also due to a limited intracystic component. This finding is similar to the study by Chen et $a l^{20}$ in which the majority of the 2400 mucoepidermoid carcinomas of the parotid were of intermediate grade. In contrast, in the study by Zhu et $a 1^{19}$ of 88 pulmonary mucoepidermoid carcinoma cases, most cases were of low-grade morphology similar to the study of 17 pulmonary mucoepidermoid carcinomas ${ }^{16}$ that also contained more low-grade $(n=10)$ than high-grade pulmonary mucoepidermoid carcinomas $(n=7)$. However, this latter study graded pulmonary mucoepidermoid carcinoma according to the WHO classification, ${ }^{21}$ which does not consider an intermediate-grade morphology. Zhu et $a 1^{19}$ graded pulmonary mucoepidermoid carcinoma according to Auclair et al. ${ }^{22}$ We graded our pulmonary mucoepidermoid carcinomas according to Brandwein et $a l^{3}$ a current grading system that is consistently used for mucoepidermoid carcinoma in our institution. Although there are some nuances between these grading systems, it becomes apparent that most pulmonary mucoepidermoid carcinomas are of low to intermediate grade and only occasional cases are of high-grade morphology.

Although the diagnosis of pulmonary mucoepidermoid carcinoma, as mucoepidermoid carcinoma in other locations, is primarily based on morphology, the distinction of high-grade pulmonary mucoepidermoid carcinoma from other entities may be problematic. Therefore, we investigated the role of immunohistochemistry as a possible adjunct to help in the diagnosis of pulmonary mucoepidermoid 
carcinoma. Bilal et $a l^{23}$ have shown that p63 is expressed in basal and myoepithelial cells of benign salivary gland tissues. p63 has been proposed to be useful in distinguishing mucoepidermoid carcinoma from other salivary gland tumor, especially acinic cell carcinoma, ${ }^{24}$ given that p63 is generally negative in acinic cell carcinomas. Moreover, Bilal et $a l^{23}$ have reported on 9 mucoepidermoid carcinomas (4 low grade, 3 intermediate grade and two high grade), all of which showed strong and diffuse staining with p63 (Table 2). There was no correlation between p63 expression and tumor grade. In cystic tumors, reactive nuclei were found in foci of intermediate or squamous cells and in basal cells at the periphery of mucous cells that lined cystic spaces. In solid tumors, sheets or islands of intermediate, squamous, and clear cells demonstrated p63 nuclear reactivity. p63 protein was absent from mucous and columnar cells. Sams et $a 1^{24}$ showed that all 24 salivary gland mucoepidermoid carcinomas showed strong expression (76-100\% reactivity) of p63. We found that in all our cases tested, p63 was expressed in the neoplastic cells. However, in the majority of our cases, p63 was expressed in $<25 \%$ of tumor cells. Similarly to the study by Bilal et $a l^{23}$ we could not identify an obvious association between p63 expression and grade, although $>50 \%$ of tumor cells stained with p63 in high-grade pulmonary mucoepidermoid carcinoma, while low- and intermediate-grade pulmonary mucoepidermoid carcinoma scores were variable. The high expression of p63 in high grade pulmonary mucoepidermoid carcinoma might be due to the large proportions of epidermoid and intermediate cells and only relatively few mucous cells in these tumors. The somewhat lower expression of p63 in our study in comparison to other mucoepidermoid carcinoma studies might be, at least in part, due to the wide variety of cases collected from multiple institutions with possible differences in fixation.

TTF-1 and napsin were negative in all cases tested, which will be further helpful to distinguish this tumor from a primary lung adenocarcinoma.

p40, a p63 isoform has recently been proposed as a more specific marker for squamous differentiation than p63. Like p63, p40 is also expressed in myoepithelial cells. To our knowledge, p40 expression has not been explored in mucoepidermoid carcinoma. We therefore compared the expression of p40 in tumor cells in pulmonary mucoepidermoid carcinoma with p63 expression. We found that in the majority of cases the expression pattern between p63 and p40 was comparable. However, in about one quarter of pulmonary mucoepidermoid carcinomas we observed a lower p40 expression score when compared with p63 and a few cases that have at least focal p63 expression were p40-negative. Therefore, p63 might be a more sensitive marker for pulmonary mucoepidermoid carcinoma than $\mathrm{p} 40$.

$\mathrm{t}(11 ; 19)(\mathrm{q} 21 ; \mathrm{p} 13)$ was identified as the major chromosomal abnormality in a large number of head and neck mucoepidermoid carcinomas. This translocation fuses the CREB Regulated Transcription Coactivator1 (CRCT1) gene and a mammalian mastermind-like 2 (MAML2) gene located on chromosomes 19p13 and 11q21, respectively. Evidence suggests that CRCT1-MAML2 fusion protein may disrupt Notch signaling. ${ }^{25}$ Furthermore, this fusion protein might mimic constitutive activation of CAMP signaling by direct activation of the cAMP response element-binding protein (CREB) ${ }^{26}$ While it was initially thought that this fusion protein might only be present in low- and intermediate-grade mucoepidermoid carcinoma, ${ }^{27}$ more recent studies identified MAML2 rearrangements also in up to $71 \%$ of high grade mucoepidermoid carcinomas. ${ }^{28,29}$ In our study, except for two cases that failed hybridization, all tested pulmonary mucoepidermoid carcinoma cases harbored MAML2 rearrangement including two high-grade pulmonary mucoepidermoid carcinomas. These results are similar to the study by Chenevert et $a l^{5}$ in which two (of 2) studied high-grade mucoepidermoid carcinomas of the head and neck had MAML2 rearrangement. Interestingly, in that study the authors had reclassified 24 of 46 originally diagnosed mucoepidermoid carcinoma cases using strict morphologic criteria and p63 expression as done in our study. In contrast, Achcar et $a l^{16}$ showed that all low-grade pulmonary mucoepidermoid carcinomas had MAML2 rearrangements while only three (of 7) high-grade pulmonary mucoepidermoid carcinomas had that rearrangement. One reason for the discrepancy between MAML2 rearrangement in high-grade mucoepidermoid carcinoma might be the strict inclusion criteria that were used in our study as well as the study by Chenevert et $a l^{5}$. In our study, in five cases, upon rereview it was felt that the morphology was not that of a pulmonary mucoepidermoid carcinoma but rather that of an adenocarcinoma, benign lesion, or spindle cell lesion. In only one of these cases, MAML2 rearrangement was identified and therefore, only this case was included in the study. The other four cases were very likely misclassified originally and were excluded. Moreover, MAML2 rearrangement has been shown to be disease-defining for mucoepidermoid carcinoma and has not been identified in any other salivary gland or non-salivary gland-type tumor. The study by Achcar et $a l^{16}$ for instance tested MAML2 rearrangement in 24 squamous cell carcinoma, 41 adenocarcinoma and 16 adenosquamous cell carcinoma, which were all negative. Therefore, MAML2 rearrangement studies might be helpful in the distinction of pulmonary mucoepidermoid carcinoma from other epithelial lung malignancies especially on small biopsies or in children in whom pulmonary mucoepidermoid carcinoma are among the most common epithelial lung malignancies and where there is special trepidation, as the surgery required is often lobectomy.

In conclusion, the majority of pulmonary mucoepidermoid carcinomas are of morphologic inter- 
mediate grade. Features helpful in distinguishing this special form of lung cancer from others include central/endo- or peribronchial location together with p63 expression, MAML2 rearrangement and lack of TTF-1 and napsin A expression. Morphologic characteristics of pulmonary mucoepidermoid carcinoma that may assist in distinguishing it from squamous cell carcinoma include scattered mucous cells, and lack of keratinization. p63 might be superior to p40 in aiding the diagnosis of pulmonary mucoepidermoid carcinoma.

\section{Disclosure/conflict of interest}

The authors declare no conflict of interest.

\section{References}

1 Welsh JH, Maxson T, Jaksic T, et al. Tracheobronchial mucoepidermoid carcinoma in childhood and adolescence: case report and review of the literature. Int J Pediatr Otorhinolaryngol 1998;45:265-273.

2 Turnbull AD, Huvos AG, Goodner JT, et al. Mucoepidermoid tumors of bronchial glands. Cancer 1971; 28:539-544.

3 Brandwein MS, Ivanov K, Wallace DI, et al. Mucoepidermoid carcinoma: a clinicopathologic study of 80 patients with special reference to histological grading. Am J Surg Pathol 2001;25:835-845.

4 Molina JR, Aubry MC, Lewis JE, et al. Primary salivary gland-type lung cancer: spectrum of clinical presentation, histopathologic and prognostic factors. Cancer 2007;110:2253-2259.

5 Chenevert J, Barnes LE, Chiosea SI. Mucoepidermoid carcinoma: a five-decade journey. Virchows Arch 2011;458:133-140.

6 Camelo-Piragua SI, Habib C, Kanumuri P, et al. Mucoepidermoid carcinoma of the breast shares cytogenetic abnormality with mucoepidermoid carcinoma of the salivary gland: a case report with molecular analysis and review of the literature. Hum Pathol 2009;40:887-892.

7 Garcia JJ, Hunt JL, Weinreb I, et al. Fluorescence in situ hybridization for detection of MAML2 rearrangements in oncocytic mucoepidermoid carcinomas: utility as a diagnostic test. Hum Pathol 2011;42:2001-2009.

8 Clauditz TS, Gontarewicz A, Wang CJ, et al. 11q21 Rearrangement is a Frequent and Highly Specific Genetic Alteration in Mucoepidermoid Carcinoma. Diagn Mol Pathol 2012;21:134-137.

9 Seethala RR, Dacic S, Cieply K, et al. A reappraisal of the MECT1/MAML2 translocation in salivary mucoepidermoid carcinomas. Am J Surg Pathol 2010;34: 1106-1121.

10 Chiosea SI, Dacic S, Nikiforova MN, et al. Prospective testing of mucoepidermoid carcinoma for the MAML2 translocation: clinical implications. Laryngoscope 2012;122:1690-1694.

11 Achcar Rde O, Nikiforova MN, Dacic S, et al. Mammalian mastermind like 2 11q21 gene rearrangement in bronchopulmonary mucoepidermoid carcinoma. Hum Pathol 2009;40:854-860.
12 Macarenco RS, Uphoff TS, Gilmer HF, et al. Salivary gland-type lung carcinomas: an EGFR immunohistochemical, molecular genetic, and mutational analysis study. Mod Pathol 2008;21:1168-1175.

13 Yousem SA, Nicholson AG. Mucoepidermoid carcinoma, In: Travis WD, Brambilla E, Mueller-Hermelink HK, Harris CC(eds) Tumours of the Lung, Pleura, Thymus and Heart. IARC Press: Lyon, France; 2004, pp 63-64.

14 Erickson-Johnson MR, Seys AR, Roth CW, et al. Carboxypeptidase M: a biomarker for the discrimination of well-differentiated liposarcoma from lipoma. Mod Pathol 2009;22:1541-1547.

15 Di Tommaso L, Foschini MP, Ragazzini $\mathrm{T}$, et al. Mucoepidermoid carcinoma of the breast. Virchows Arch 2004;444:13-19.

16 Achcar Rde O, Nikiforova MN, Dacic S, et al. Mammalian mastermind like 2 11q21 gene rearrangement in bronchopulmonary mucoepidermoid carcinoma. Hum Pathol 2009;40:854-860.

17 Riedlinger WF, Hurley MY, Dehner LP, et al. Mucoepidermoid carcinoma of the skin: a distinct entity from adenosquamous carcinoma: a case study with a review of the literature. Am J Surg Pathol 2005;29:131-135.

18 Roden AC, Erickson-Johnson MR, Yi ES, et al. Analysis of MAML2 rearrangement in mucoepidermoid carcinoma of the thymus. Hum Pathol 2013;44: 2799-2805.

19 Zhu F, Liu Z, Hou Y, et al. Primary salivary gland-type lung cancer: clinicopathological analysis of 88 cases from china. J Thorac Oncol. 2013;8:1578-1584.

20 Chen MM, Roman SA, Sosa JA, et al. Histologic grade as prognostic indicator for mucoepidermoid carcinoma: A population-level analysis of 2400 patients. Head Neck 2014;36:158-163.

21 Travis WD, Brambilla E, Muller-Hermelink HK, et al. Pathology and genetics of tumors of the lung, pleura, thymus and heart, In: Kleihues P, Sobin LH (eds) WHO Classification of Tumors, 2nd edn. IARC Press: Lyon, France; 2004, pp 63-64.

22 Auclair PL, Goode RK, Ellis GL. Mucoepidermoid carcinoma of intraoral salivary glands. Evaluation and application of grading criteria in 143 cases. Cancer 1992;69:2021-2030.

23 Bilal H, Handra-Luca A, Bertrand JC, et al. P63 is expressed in basal and myoepithelial cells of human normal and tumor salivary gland tissues. J Histochem Cytochem 2003;51:133-139.

24 Sams RN, Gnepp DR. P63 expression can be used in differential diagnosis of salivary gland acinic cell and mucoepidermoid carcinomas. Head Neck Pathol 2013;7:64-68.

25 Tonon G, Modi S, Wu L, et al. t(11;19)(q21;p13) translocation in mucoepidermoid carcinoma creates a novel fusion product that disrupts a Notch signaling pathway. Nat Genet 2003;33:208-213.

$26 \mathrm{Wu} \mathrm{L}$, Liu J, Gao P, et al. Transforming activity of MECT1-MAML2 fusion oncoprotein is mediated by constitutive CREB activation. Embo J 2005;24:2391-2402.

27 Okabe M, Miyabe S, Nagatsuka H, et al. MECT1MAML2 fusion transcript defines a favorable subset of mucoepidermoid carcinoma. Clin Cancer Res. 2006;12: 3902-3907.

28 Seethala RR, Dacic S, Cieply K, et al. A reappraisal of the MECT1/MAML2 translocation in salivary mucoepidermoid carcinomas. Am J Surg Pathol. 2010;34:1106-1121. 
29 Tirado Y, Williams MD, Hanna EY, et al. CRTC1/MAML2 fusion transcript in high grade mucoepidermoid carcinomas of salivary and thyroid glands and Warthin's tumors: implications for histogenesis and biologic behavior. Genes Chromosomes Cancer 2007;46:708-715.

30 Whithaus K, Fukuoka J, Prihoda TJ, et al. Evaluation of napsin A, cytokeratin 5/6, p63, and thyroid transcription factor 1 in adenocarcinoma versus squamous cell carcinoma of the lung. Arch Pathol Lab Med. 2012; 136:155-162.

31 Bishop JA, Teruya-Feldstein J, Westra WH, et al. p40 (DeltaNp63) is superior to p63 for the diagnosis of pulmonary squamous cell carcinoma. Mod Pathol. 2012;25:405-415.

32 Nonaka D. A study of DeltaNp63 expression in lung non-small cell carcinomas. Am J Surg Pathol. 2012;36: 895-899.

33 Rekhtman N, Ang DC, Sima CS, et al. Immunohistochemical algorithm for differentiation of lung adeno- carcinoma and squamous cell carcinoma based on large series of whole-tissue sections with validation in small specimens. Mod Pathol. 2011;24:1348-1359.

34 Cadioli A, Rossi G, Costantini M, et al. Lung cancer histologic and immunohistochemical heterogeneity in the era of molecular therapies: analysis of 172 consecutive surgically resected, entirely sampled pulmonary carcinomas. Am J Surg Pathol 2014;38: 502-509.

35 Ordonez NG. A word of caution regarding napsin A expression in squamous cell carcinomas of the lung. Am J Surg Pathol 2012;36:396-401.

36 Turner BM, Cagle PT, Sainz IM, et al. Napsin A, a new marker for lung adenocarcinoma, is complementary and more sensitive and specific than thyroid transcription factor 1 in the differential diagnosis of primary pulmonary carcinoma: evaluation of 1674 cases by tissue microarray. Arch Pathol Lab Med 2012;136: 163-171. 